\title{
The hereditary ataxias: Where are we now? Four decades of local research
}

\author{
D C Smith, ${ }^{1,2}$ MSc, PhD; L J Greenberg, ${ }^{1} \mathrm{PhD} ;$ A Bryer, ${ }^{2} \mathrm{MB}$ ChB, FCP(SA), MMed (Neurology), FC Neurology (SA), PhD \\ ${ }^{1}$ Division of Human Genetics, Institute of Infectious Disease and Molecular Medicine, Department of Pathology, Faculty of Health Sciences, \\ University of Cape Town, South Africa \\ ${ }^{2}$ Division of Neurology, Department of Medicine, Faculty of Health Sciences, University of Cape Town, Groote Schuur Hospital, Cape Town, \\ South Africa
}

\section{Corresponding author: D C Smith (danielle@gsmith.co.za)}

\begin{abstract}
The hereditary ataxias have been studied at the University of Cape Town for more than 40 years, following from initial clinical investigations by Beighton and colleagues in the early 1970s. This group of inherited disorders is characterised by progressive neurodegeneration and associated symptoms, including the inability to coordinate movement. Following initial local and international linkage studies, and the discovery of the genes responsible for the key dominant and recessive inherited ataxias in the 1990s, a local molecular testing service was established at Groote Schuur Hospital. More than 1600 individuals have been referred through this testing service (now offered by the National Health Laboratory Service), leading to the molecular diagnosis of 253 families with spinocerebellar ataxia types 1, 2, 3, 6 or 7, and 30 families with Friedreich's ataxia. This is likely to be an under-representation of the number of South Africans affected with hereditary ataxia, and future research efforts will focus on increasing the awareness of this group of disorders, both locally and throughout the rest of Africa. Next-generation technologies will be beneficial in identifying additional genes underlying inherited ataxia in indigenous patients to enable more appropriate management and treatment of individuals with molecularly undiagnosed forms of the disease.
\end{abstract}

S Afr Med J 2016;106(6 Suppl 1):S38-S41. DOI:10.7196/SAMJ.2016.v106i6.10989

The hereditary ataxias are characterised by progressive neurodegeneration, leading to permanent disability. Symptoms of hereditary ataxia typically include a progressive incoordination of gait, hands and speech. The inability to coordinate movement has a significant impact on the quality of life of patients, as well as their family members and caregivers. Furthermore, palliative care and management of these individuals comes at a significant cost. Ataxia may be inherited in an autosomal dominant, autosomal recessive or $\mathrm{X}$-linked manner. ${ }^{[1]}$ The most common forms of dominant inherited ataxia are the spinocerebellar ataxias (SCAs) type 1, 2, 3, 6 and 7, caused by the expansion of a CAG repeat tract beyond a pathogenic threshold. Friedreich's ataxia (FRDA) is the most common recessive ataxia, resulting from the expansion of a GAA repeat within the FXN gene. ${ }^{[2]}$

The global epidemiology of the hereditary ataxias is largely unknown, but a meta-analysis of prevalence studies has led to the estimation that an average of 2.7 per $10^{5}$ individuals are affected with autosomal dominant hereditary ataxia. ${ }^{[3]}$ Given the current African population estimate of 1.11 billion, approximately 30000 Africans may be affected with one of these disorders. Reports on the prevalence of the inherited ataxias on the African continent, particularly sub-Saharan Africa, have been extremely scarce. Many of the existing reports in the literature are not representative of a large geographical area, but rather of isolated cases and families.

\section{The past}

In the 1970s, a large family of coloured individuals with autosomal dominant ataxia was identified in the Western Cape region of South Africa (SA). Of concern at the time was that affected individuals often had transmitted the gene before they were aware of the condition. There were no genetic tests available at that time. A study was undertaken to determine whether or not there were early presymptomatic clinical markers for the condition that could be of value for genetic counselling. In an early publication in Brain by Philcox et al., ${ }^{[4]}$ it was documented that defective optokinetic nystagmus and absent or abnormal ocular-vestibular responses in association with normal cochlear function were an early manifestation of the disorder in this family. ${ }^{[4]}$ Additional families with adult-onset autosomal dominant ataxia were identified in the neurology units at the two academic tertiary hospitals in that region, Groote Schuur Hospital (GSH) and Tygerberg Hospital. Subsequent to that early study, a large prospective population-based study was undertaken by Alan Bryer as part of his $\mathrm{PhD}$ project. Pedigrees of individual families were carefully documented and both affected and unaffected individuals were assessed. Appropriate electrophysiological testing and magnetic resonance imaging brain scans were done to phenotype selected affected patients. In the early years, an initial conventional linkage study was undertaken in the largest family, and this showed linkage to the HLA locus on chromosome $6 \cdot{ }^{[5]} \mathrm{A}$ multi-locus linkage analysis confirmed the assignment of a locus for the disease distal to HLA. In 1991 the gene for SCA type 1 (SCA1) was identified and shown to be caused by an expanded unstable CAG repeat within the gene on chromosome $6 \mathrm{p}^{[6,7]}$ A collaboration with Huda Zoghbi from Minnesota confirmed that the local large family had the SCA1 mutation. A haplotype-based study later confirmed a SCA1 founder effect in the SA coloured population. ${ }^{[8]}$ In the era of molecular genetics that followed, further mutations were identified for the dominant ataxias. Consequent to the population-based study, a predictive testing service for the familial ataxias was established at GSH. This study was extended to the other provinces and blood samples were received from affected kindred from all over SA since 1994.

\section{The present}

To date, approximately 1600 individuals from 1280 families have been referred to the Division of Human Genetics/National 
Health Laboratory Service (NHLS) for testing for one or more of the SCAs. Roughly $20 \%$ of these individuals have received a confirmed molecular diagnosis of SCA types 1, 2, 3, 6 or 7. The distribution of families diagnosed with one of the dominant SCAs has not changed remarkably since the first report on 44 families in 2003 (Fig. 1, left panel, from Bryer et al. ${ }^{[9]}$ ), although a larger proportion of families have now been diagnosed with SCA2 (Fig. 1, right panel, based on 253 families). SCA1 remains the most commonly diagnosed form of dominant ataxia in SA. SCA3 is commonly reported as the most prevalent form of SCA globally. However, only 14 SA families have been diagnosed with SCA3 since the initiation of testing. The high prevalence of SCA7 in the SA black African population is another unique feature, which has led to numerous local research projects (see Watson et al. elsewhere in this edition).

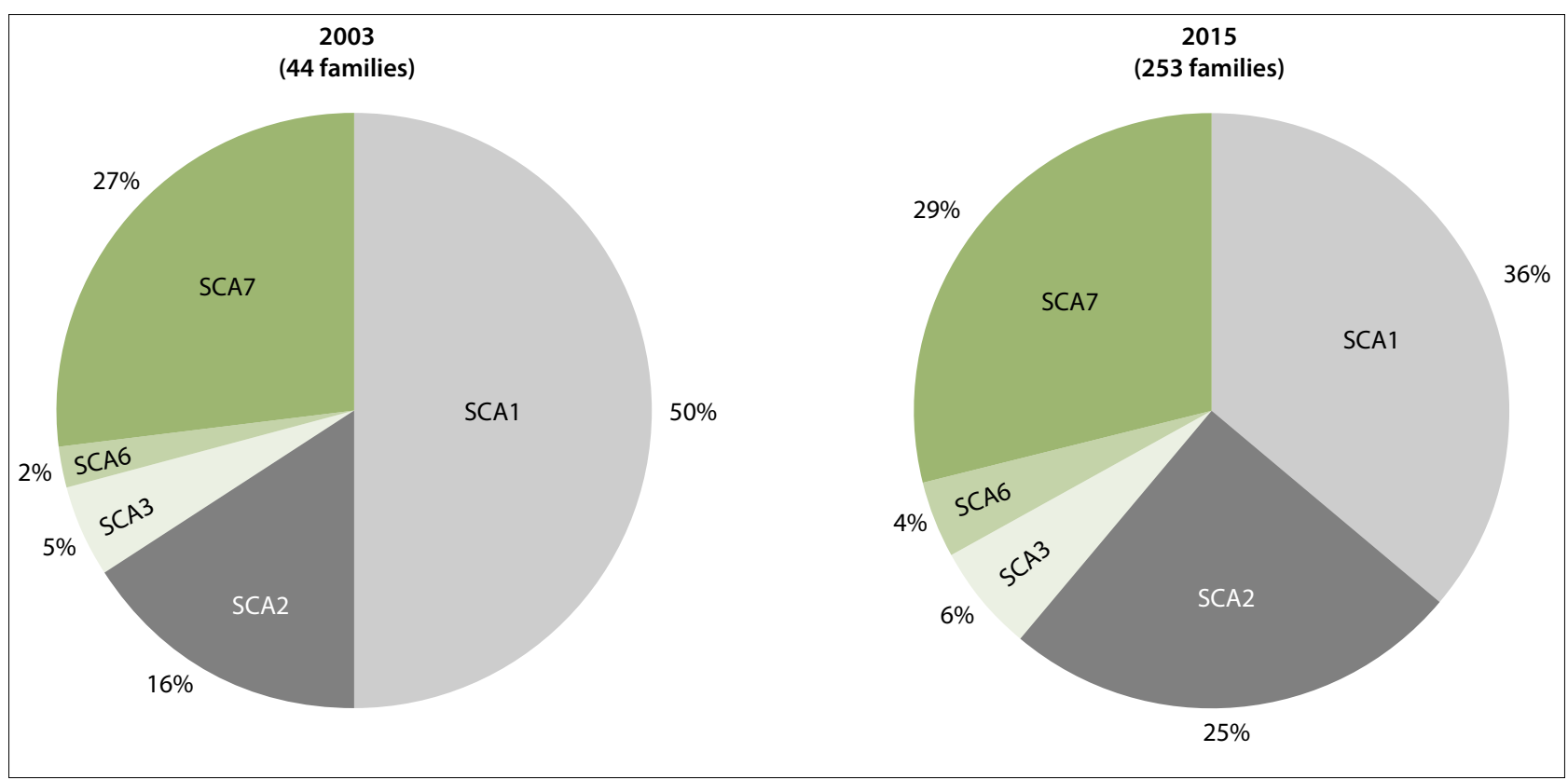

Fig. 1. Distribution of SA families diagnosed with SCA types 1, 2, 3, 6 or 7 from the 1990s until 2003 (left) and 2015 (right).

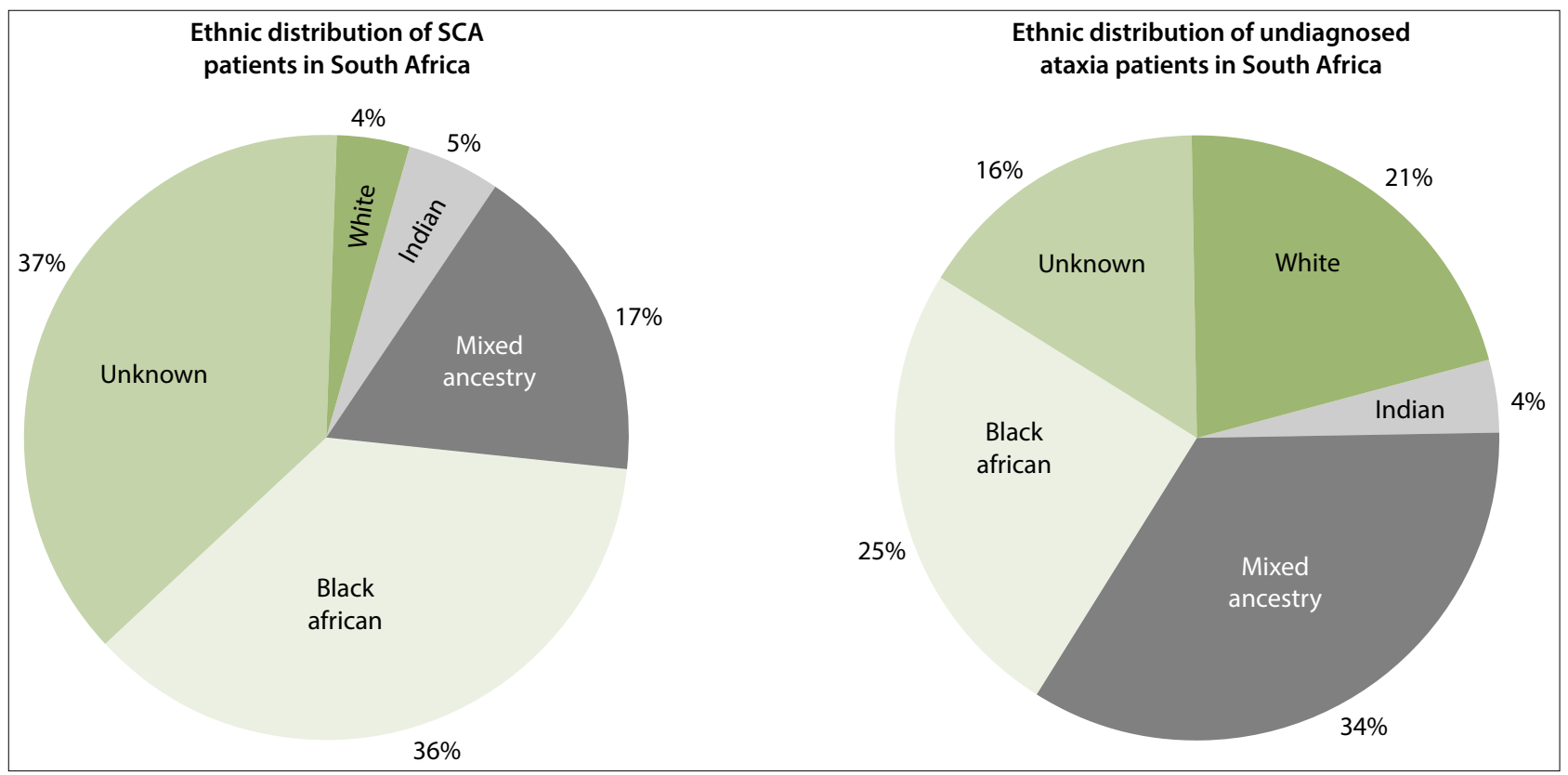

Fig. 2. Population distributions of SA SCA patients (left) and molecularly undiagnosed individuals with ataxia (right).
The information supplied with molecular diagnostic testing referrals rarely contains detailed biographical or clinical information. However, we estimate that at least $50 \%$ of the individuals diagnosed with SCA are black African or coloured (a population unique to SA, containing an admixture of European, Khoisan, Asian and black African ancestries) (Fig. 2, left panel). Since these population groups are unique to the sub-Saharan African region, this figure indicates that inherited forms of ataxia are prevalent in 'indigenous' African populations, and not only in individuals of European descent. Similarly, over $50 \%$ of ataxia patients who remain molecularly undiagnosed, are black African or coloured (Fig. 2, right panel).

Additionally, over a period of 15 years of testing, approximately 327 individuals from 266 families have had molecular testing for recessive FRDA. Thirty-seven of these individuals (from 30 families) had molecularly confirmed FRDA. To the best of our knowledge, 


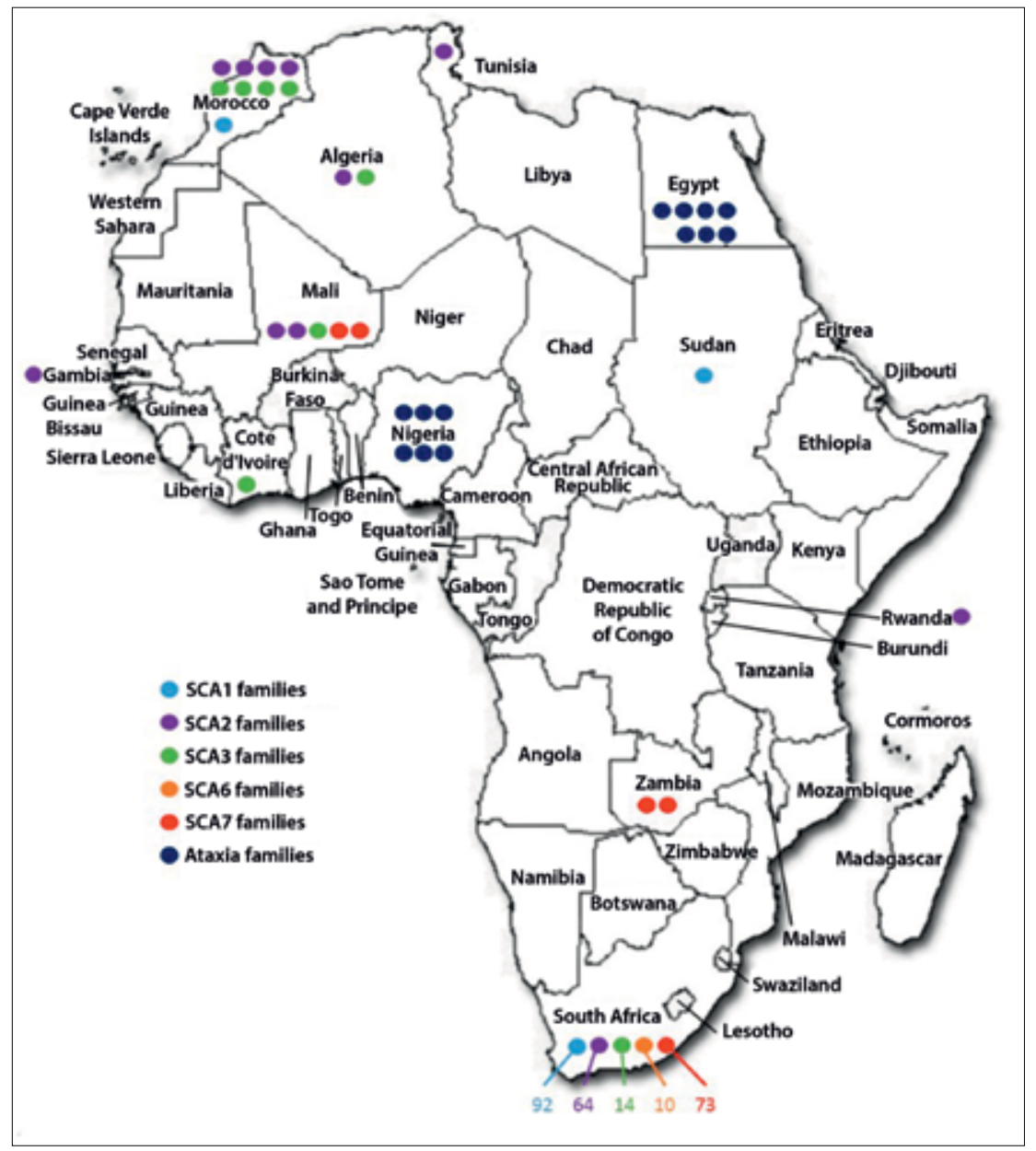

Fig. 3. Published reports of African families with inherited ataxia (SCA types 1, 2, 3, 6, 7 or unidentified 'inherited ataxia'). Circles represent the number of described families per country ${ }^{[10-21]}$

no individuals of indigenous black African ethnic origin have been given a confirmed molecular diagnosis of FRDA. The vast majority of confirmed FRDA patients in SA are of European ancestry, along with a single family of Indian origin.

We have shown that inherited forms of ataxia are prevalent within African ethnic groups. However, published reports on the occurrence of these disorders on the African continent have been extremely scarce (Fig. 3). Although the expected prevalence of the inherited ataxias in Africa is significantly lower than other widespread diseases such as HIV and tuberculosis, the potential impact of future research into these disorders cannot be ignored.

\section{The future}

While almost 1600 individuals had been referred to our centre for molecular testing over a 27 -year period, only $21 \%$ have received a confirmed molecular diagnosis. Firstly, this is likely to be an under-representation of the number of South Africans affected with the SCAs, since financial constraints and
In cases of families with a strong family history, but with an unknown genetic cause, it may be beneficial to employ next-generation technologies such as whole genome or exome sequencing. Németh et al. ${ }^{[22]}$ demonstrated the utility of this approach by using nextgeneration sequencing in an attempt to determine the underlying mutations in 50 patients with familial or early-onset ataxia who had previously been tested for SCA types 1, 2, 3, 6, 7 and FRDA. Targeted capture performed on 117 candidate genes revealed 13 pathogenic mutations (nine of which were novel) in the patient cohort, indicating that the inclusion of this strategy may be advantageous in diagnostic laboratories. However, it should be noted that this method may be accompanied by new challenges, such as increased cost and difficulty in interpreting the pathogenicity of new variants, therefore it may be some time before it is adopted into routine practice in resource-limited countries.

As part of future studies, we will contact neurologists throughout sub-Saharan Africa, requesting referrals of individuals with suspected forms of inherited ataxia. These patients will be tested for SCA types 1, 2, 3, 6, 7 and FRDA. We will then employ next generation sequencing technologies to identify the genetic causes of ataxia in patients without mutations at the previously mentioned loci. By achieving these aims, we hope to develop a project called 'Ataxia Africa' - a network of clinicians and scientists working together to identify and diagnose patients with ataxia on the African continent.

number of clinical referrals from low-income and rural areas. Secondly, it is of concern that almost $80 \%$ of the referred individuals did not receive a molecular diagnosis. While this may be due to poor or unwarranted clinical referrals, it is possible that a proportion of these patients are affected with an additional type of SCA that does not form part of the current testing panel (SCA types 1, 2, 3, 6, 7, 12, 17 and FRDA). Clinical or familial information rarely accompanies biological samples, therefore it is unknown whether these undiagnosed individuals come from families with multiple affected members. It is also not known whether any particular population group is over-represented in this 'undiagnosed' cohort, since the ethnicities of only a small proportion of this group is known. Studies undertaken in other countries may be useful in determining whether other types of ataxia should be included in the testing panel, but the SA population is highly heterogeneous and unique, and no other broad SCA-based epidemiological investigations have been completed in other African countries.

\section{Conclusion}

To the best of our knowledge, the NHLS laboratory at GSH in Cape Town is the only laboratory offering a molecular diagnostic service for the inherited ataxias on the African continent. Additionally, the Division of Human Genetics at the University of Cape Town (UCT) is the only research group in Africa investigating this group of disorders, spanning a period of almost five decades. A large number of individuals and families would benefit from increased awareness, molecular testing and research for the inherited ataxias in Africa. A confirmed molecular diagnosis can enable more appropriate clinical management of the affected individuals, and family members can be counselled with regard to their own risk, as well as the risk for current and future offspring. Additionally, individuals with a confirmed diagnosis may be eligible for future clinical trials and therapeutic interventions. 
Acknowledgements. The authors wish to thank the following for their long-standing contributions to ataxia research at UCT: Dr Lauren Watson, Ms Alina Esterhuizen, Dr Fiona Baine, Dr Janine Scholefield, Associate Professor Jeannine Heckmann, Professor Matthew Wood. The authors also acknowledge numerous funding sources, including the National Research Foundation, Medical Research Council, UCT, John Fell Fund and Ataxia UK.

\section{References}

1. Bird TD. Hereditary ataxia overview. In: Pagon RA, Adam MP, Ardinger HH, et al, editors. GeneReviews. Seattle: University of Washington, 1993.

2. Campuzano V, Montermini L, Molto MD, et al. Friedreich's ataxia: Autosomal recessive disease caused by an intronic GAA triplet repeat expansion. Science 1996;271(5254):1423-1427. DOI:10.1126/ by an intronic GAA
science. 271.5254 .1423

3. Ruano L, Melo C, Silva M, Coutinho P. The global epidemiology of hereditary ataxia and spastic paraplegia: A systematic review of prevalence studies. Neuroepidemiology 2014;42(3):174-183. DOI: $10.1159 / 00035880$

4hilcox DV, Sellars SL, Pamplett R, Beighton P. Vestibular dysfunction in hereditary ataxia. Brain 1975;98(2):309-316. DOI:10.1093/brain/98.2.309

5. Bryer A, Martell R, Du Toit E, Beighton P. Adult onset spinocerebellar ataxia linked to HLA in a South African kindred of mixed ancestry. Tissue Antigens 1992;40(3):111-115. DOI:10.1111/j.1399-0039.1992.tb02101.x

6. Zoghbi HY, Jodice C, Sandkuijl LA, et al. The gene for autosomal dominant spinocerebellar ataxia (SCA1) maps telomeric to the HLA complex and is closely linked to the D6S89 locus in three large kindreds. Am J Hum Genet 1991;49(1):23-30.

7. Orr HT, Chung M, Banfi S, et al. Expansion of an unstable trinucleotide CAG repeat in spinocerebellar ataxia type 1. Nat Genet 1993;4(3):221-226. DOI:10.1038/ng0793-221
8. Ramesar RS, Bardien S, Beighton P, Bryer A. Expanded CAG repeats in spinocerebellar ataxia (SCA1) segregate with distinct haplotypes in South African families. Hum Genet 1997;100(1):131-137. DOI:10.1007/s004390050478

9. Bryer A, Krause A, Bill P, et al. The hereditary adult-onset ataxias in South Africa. J Neurol Sci 2003;216(1):47-54. DOI:10.1016/S0022-510X(03)00209-0

10. Aiyesimoju $\mathrm{AB}$, Osuntokun BO, Bademosi O, Adeuja AO. Hereditary neurodegenerative disorders in Nigerian Africans. Neurology 1984;34(3):361-362. DOI:10.1212/WNL.34.3.361

11. Dürr A, Stevanin G, Cancel G, et al. Spinocerebellar ataxia 3 and Machado-Joseph disease: Clinical, molecular, and neuropathological features. Ann Neurol 1996;39(4):490-499. DOI:10.1002/ana.41039041 2. Benomar A, Meggouh F, Didierjean O, et al. Autosomal dominant cerebellar ataxia type I in Morocco: Presence of the SCA1 and SCA3/MJD mutations. Eur J Neurol 1996;3(4):369-372. DOI:10.1111/j.1468-1331.1996.tb00231.x

13. Cancel G, Dürr A, Didierjean O, et al. Molecular and clinical correlations in spinocerebellar ataxia 2: A study of 32 families. Hum Mol Genet 1997;6(5):709-715. DOI:10.1093/hmg/6.5.709

14. Modi G, Modi M, Martinus I, Rodda J, Saffer D. The clinical and genetic characteristics of spinocerebellar ataxia type 7 (SCA 7) in three Black South African families. Acta Neurol Scand 2000;101(3):177-182. DOI:10.1034/j.1600-0404.2000.101003177.x

15. Traoré M, Coulibaly T, Meilleur KG, et al. Clinical and genetic analysis of spinocerebellar ataxia in Mali. Eur J Neurol 2011;18(10):1269-1271. DOI:10.1111/ji.1468-1331.2011.03376.x

6. (Riyadh) 2002;7(2):83-85

17. Mutesa L, Uwineza A, Hellin A, et al. A survey of genetic diseases in Rwanda. 2013;68(3):5-17.

8. Smith D, Bryer A, Watson L, Greenberg L. Inherited polyglutamine spinocerebellar ataxias in South Africa. S Afr Med J 2012;102(8):683-686

19. El Tallawy HN, Farghaly WM, Rageh TA, et al. Epidemiology of major neurological disorders project in Al Kharga district, New Valley, Egypt. Neuroepidemiology 2010;35(4):291-297. DOI:10.1159/000320240

20. Smith DC, Atadzhanov M, Mwaba M, Greenberg LJ. Evidence for a common founder effect amongst South African and Zambian individuals with Spinocerebellar ataxia type 7. J Neurol Sci 2015;354(1-2): 75-78. DOI:10.1016/j.jns.2015.04.053

21. Almaguer-Mederos LE, Sarr L, Abascal JV, et al. Spinocerebellar ataxia type 2 in The Gambia: A case report. J Neurol Sci 2015;349(1-2):269-271. DOI:10.1016/j.jns.2015.01.027

22. Németh $\mathrm{AH}$, Kwasniewska $\mathrm{AC}$, Lise $\mathrm{S}$, et al. Next generation sequencing for molecular diagnosis of neurological disorders using ataxias as a model. Brain 2013;136(Pt 10):3106-3118. DOI:10.1093/brain/awt236 